\title{
STUDY OF CLINICAL AND DEMOGRAPHIC PROFILE OF OCULAR TRAUMA AND ITS EFFECT ON VISUAL OUTCOME
}

\author{
Inderjit Kaur 1 , Prem Pal Kaur², Atish Pradhan ${ }^{3}$, Bhav Karan Singh Bal 4 , Piyush Goyal5, Nimrata Bajaj ${ }^{6}$ \\ 1 Professor, Department of Ophthalmology, Government Medical College, Amritsar. \\ ${ }^{2}$ Associate Professor, Department of Ophthalmology, Government Medical College, Amritsar. \\ 3 Junior Resident, Department of Ophthalmology, Government Medical College, Amritsar. \\ 4Junior Resident, Department of Orthopaedics, SGRDIMSR, Amritsar. \\ 5Junior Resident, Department of Ophthalmology, Government Medical College, Amritsar. \\ 6Junior Resident, Department of Ophthalmology, Government Medical College, Amritsar.
}

\begin{tabular}{l}
\hline ABSTRACT \\
BACKGROUND \\
Ocular trauma is an important preventable cause of visual morbidity, especially among younger population in developing \\
countries. It constitutes a significant cause of monocular blindness. \\
Aims- To study the demographic and clinical profile of ocular trauma and its effect on visual outcome.
\end{tabular}

\section{MATERIALS AND METHODS}

This prospective study was conducted on 200 patients visiting Regional Institute of Ophthalmology, Govt. Medical College, Amritsar with ocular trauma. All patients above 5 years of age were included in the study. Patients with comorbid eye diseases affecting the visual acuity were excluded from the study. Data obtained was tabulated and analysed statistically.

\section{RESULTS}

Out of 100 patients, 46\% were between 16 - 35 years of age. Males (88\%) were predominantly affected. Road side accident (30\%) was found to be the most common cause of injury; 71 patients presented within 5 days of injury. Closed globe injury (81\%) was more common than open globe. Conjunctiva (80 patients) was the most commonly involved ocular structure followed by eyelids ( 60 patients). The visual acuity observed in the affected eye at the time of admission was between $6 / 18-6 / 6$ in $44 \%$ of patients followed by $<3 / 60$ in $25 \%$.

\section{CONCLUSION}

Road side accident is the most common cause of ocular trauma. Younger age group and males are predominantly affected. Closed globe is the common type of injury and conjunctiva is the mostly affected ocular structure.

\section{KEYWORDS}

Ocular Trauma, Conjunctiva, Visual Acuity.

HOW TO CITE THIS ARTICLE: Kaur I, Kaur PP, Pradhan A, et al. Study of clinical and demographic profile of ocular trauma and its effect on visual outcome. J. Evolution Med. Dent. Sci. 2017;6(37):3038-3045, DOI: 10.14260/Jemds/2017/655

\section{BACKGROUND \\ Ocular trauma is one of the commonest causes of ophthalmic morbidity and monocular blindness in all parts of the world. They have a significant impact on an individual and society in terms of sufferings, medical cost and loss of productivity.(1) \\ The eyes occupy only $0.1 \%$ of the total and $0.27 \%$ of the anterior body surface area; however, ocular trauma is far more commonly seen than what these small figures might indicate. Out of all types of ocular emergencies, ocular trauma is by far the commonest constituting nearly $75 \%$ of all ocular \\ The World Health Organisation program for the prevention of blindness, research indicated that there are approximately 55 million eye injuries per year that restrict activities for more than one day.(4) \\ In India, there are more than 50 million blind people and this number increases by about 3.8 million per year. Amongst the total number of blind cases, 1.2 percent is contributed by injuries which are preventable.(5) \\ Nearly, $90 \%$ of eye injuries can be prevented by relatively simple measures.(6)} emergencies. ${ }^{(2)}$

There are approximately 1.6 million people blind due to injuries, additional 2.3 million cases have bilateral low visual disability and 19 million have unilateral visual impairment.(3)

Financial or Other, Competing Interest: None.

Submission 25-03-2017, Peer Review 24-04-2017,

Acceptance 01-05-2017, Published 08-05-2017.

Corresponding Author:

Dr. Prem Pal Kaur,

\#333-A,

Medical Enclave,

Amritsar-143001.

E-mail:ppkbal@gmail.com

DOI: $10.14260 /$ jemds $/ 2017 / 655$

\section{(c) (i) $(5)$}

\section{Classification of Ocular Trauma (Birmingham Eye Trauma Terminology)}

1. Closed Globe

(a) Contusion.

(b) Lamellar Laceration (Partial thickness wound in eye wall).

\section{Open Globe}
(a) Penetrating (Entrance wound only).
(b) Perforating (Entrance and exit wound).
(c) IOFB (Intraocular Foreign Body).
(d) Globe Rupture.(7)

Looking at the present scenario of working pattern and visual demands of patients and the use of sophisticated 
instruments, it has become mandatory on the part of an ophthalmologist to identify the various ocular structures involved due to trauma, which may vary in severity and provide best vision if managed at its earliest. It is essential to study not only recent injuries, but also study the eyes which have sustained an injury at variable time intervals earlier.

\section{Aims}

To study the demographic and clinical pattern of ocular trauma and to assess its effect on visual acuity.

\section{MATERIALS AND METHODS}

A prospective, non-comparative study was conducted in Regional Institute of Ophthalmology, Amritsar, Punjab, India. 100 patients were studied regarding mode, type, severity of injury. The study was conducted after approval from thesis and ethical committee. Informed consent was taken. Patient had the right to opt out of the trial at any time during the course of study without having to give the reasons for doing so.

\section{Inclusion Criteria}

All patients above 5 years of age with ocular trauma were included in the study.

\section{Exclusion Criteria}

1. Children less than 5 years.

2. Comorbid eye diseases affecting visual acuity.

When a patient presented with a history of ocular trauma, following findings were recorded

1. General history.

2. Detailed history of traumatic event.

3. General physical examination.

4. Recording of presenting vision (Snellen's visual acuity).

5. External examination of eye (adnexa).

6. Ocular motility.

7. Pupils (Size, shape, symmetry, reaction to light).

8. Anterior segment (Conjunctiva, cornea, anterior chamber, iris, lens).

9. Posterior segment (Vitreous, retina, macula, choroid and sclera).

10. Intraocular pressure. (Measured by non-contact tonometer or digitally as feasible).

Ancillary Diagnostic Tests were used when required which Included

1. Plain x-ray.

2. B-scan.

3. CT scan.

4. MRI scan.

Patient was managed medically or surgically as required.

Final best corrected visual acuity was recorded using Snellen's chart following the treatment.

\section{RESULTS}

Out of 100 patients, 88 were males and 12 were females. (Table 1) 19 were between the age group 6 - 15 years, 23 each were between the age group $16-25$ years and $26-35$ years, 12 were between the age group 36 - 45 years, 14 were between the age group 46 - 55 years and 9 were more than 55 years (Table 2 ).

\begin{tabular}{|c|c|c|}
\hline Sex & No. of Patients & \% Age \\
\hline Male & 88 & 88.0 \\
\hline Female & 12 & 12.0 \\
\hline Total & $\mathbf{1 0 0}$ & $\mathbf{1 0 0 . 0}$ \\
\hline \multicolumn{2}{|c|}{ Table 1. Showing Sex Incidence } \\
\hline
\end{tabular}

\begin{tabular}{|c|c|c|}
\hline Age Group (Years) & $\begin{array}{c}\text { No. of } \\
\text { Patients }\end{array}$ & \% Age \\
\hline $6-15$ & 19 & 19.0 \\
\hline $16-25$ & 23 & 23.0 \\
\hline $26-35$ & 23 & 23.0 \\
\hline $36-45$ & 12 & 12.0 \\
\hline $46-55$ & 14 & 14.0 \\
\hline$>55$ & 9 & 9.0 \\
\hline \multicolumn{2}{|c|}{ Total Table 2. Showing Age Incidence } \\
\hline
\end{tabular}

\begin{tabular}{|c|c|c|}
\hline Eye & No. of Patients & \% Age \\
\hline OD (Right) & 49 & 49.0 \\
\hline OS (Left) & 49 & 49.0 \\
\hline OU (Both) & 2 & 2.0 \\
\hline Total & $\mathbf{1 0 0}$ & $\mathbf{1 0 0 . 0}$ \\
\hline \multicolumn{2}{|c|}{ Table 3. Showing Eye Involved } \\
\hline
\end{tabular}

Table 3 shows that out of 100 patients 49 patients had an injury in right eye, 49 patients had in left eye and 2 had in both eyes. Bilateral injuries were caused by roadside accident.

\begin{tabular}{|c|c|c|}
\hline Occupation & No. of Patients & \% Age \\
\hline Student & 34 & 34.0 \\
\hline Labour & 21 & 21.0 \\
\hline Farmer & 13 & 13.0 \\
\hline Industry & 13 & 13.0 \\
\hline Housewife & 8 & 8.0 \\
\hline Sedentary & 7 & 7.0 \\
\hline Nil & 4 & 4.0 \\
\hline Total & 100 & 100.0 \\
\hline \multicolumn{3}{|c|}{$\begin{array}{l}\text { Table 4. Showing Occupation of Patients with Ocular } \\
\text { Trauma }\end{array}$} \\
\hline
\end{tabular}

Table 4 shows that out of 100 patients 34 were students, 21 were labourers, 13 each were farmers and industrial workers, 8 were housewives, 7 were sedentary workers and 4 were unemployed.

\begin{tabular}{|c|c|c|}
\hline Source/Place & No. of Patients & \% Age \\
\hline RSA & 30 & 30.0 \\
\hline Home & 22 & 22.0 \\
\hline Assault & 20 & 20.0 \\
\hline Work & 15 & 15.0 \\
\hline Sports & 13 & 13.0 \\
\hline Total & $\mathbf{1 0 0}$ & $\mathbf{1 0 0 . 0}$ \\
\hline \multicolumn{2}{|c|}{ Table 5. Showing Place of Injury } \\
\end{tabular}


Table 5 shows that out of 100 patients, majority (30 patients) were involved in road side accidents, 22 patients had home-related injuries, 20 were assaulted, 15 were injured at work place and 13 while playing.

\begin{tabular}{|c|c|c|}
\hline Object & No. of Patients & \% Age \\
\hline RSA & 30 & 30.0 \\
\hline Wood & 12 & 12.0 \\
\hline Stick & 10 & 10.0 \\
\hline Fist & 8 & 8.0 \\
\hline Stone & 6 & 6.0 \\
\hline Metal & 6 & 6.0 \\
\hline Rod & 5 & 5.0 \\
\hline Table & 4 & 4.0 \\
\hline Cracker & 4 & 4.0 \\
\hline Glass & 2 & 2.0 \\
\hline Door & 2 & 2.0 \\
\hline Knife & 2 & 2.0 \\
\hline Blade & 2 & 2.0 \\
\hline Sand & 1 & 1.0 \\
\hline Shoe & 1 & 1.0 \\
\hline Pipe & 1 & 1.0 \\
\hline Pillar & 1 & 1.0 \\
\hline Oil & 1 & 1.0 \\
\hline Nail & 1 & 1.0 \\
\hline Tennis Ball & 1 & 1.0 \\
\hline Total & 100 & 100.0 \\
\hline \multicolumn{3}{|c|}{ Table 6. Showing Mode of Injury } \\
\hline
\end{tabular}

Table 6 shows that out of 100 patients 30 had Road Side Accidents (RSA), 12 had an injury with wood, 10 with a stick, 8 with a fist, 6 each with stone and metal, 5 with rod, 4 each with table and cracker, 2 each with glass door, knife and blade whereas 1 each with sand, shoe, pipe, pillar, oil, nail and tennis ball.

\begin{tabular}{|c|c|c|}
\hline Time Interval & No. of Patients & \% Age \\
\hline$<24$ hours & 27 & 27.0 \\
\hline 1 - 5 days & 44 & 44.0 \\
\hline 6 days - 1 month & 17 & 17.0 \\
\hline month - 12 months & 9 & 9.0 \\
\hline$>12$ months & 3 & 3.0 \\
\hline Total & $\mathbf{1 0 0}$ & $\mathbf{1 0 0 . 0}$ \\
\hline \multicolumn{2}{|c|}{ Table 7. Showing Time of Presentation } \\
\hline
\end{tabular}

Table 7 shows that out of 100 patients 27 presented within 24 hours, 44 patients consulted between 1 - 5 days, 17 between 6 days - 1 month, 9 between 1 month - 12 months and 3 patients presented after 12 months.

\begin{tabular}{|c|c|c|}
\hline Type of Injury & No. of Patients & \% Age \\
\hline Closed & 81 & 81.0 \\
\hline Open & 19 & 19.0 \\
\hline Total & $\mathbf{1 0 0}$ & $\mathbf{1 0 0 . 0}$ \\
\hline \multicolumn{2}{|c|}{ Table 8. Showing Type of Injury } \\
\hline
\end{tabular}

Table 8 shows that out of 100 patients, 81 had closed globe injuries, whereas 19 had open globe injuries.

Table 9 shows that out of 100 patients included in our study periorbital lesions were seen in 41 patients, eyelids were involved in 66 patients, conjunctiva in 79, cornea in 43, anterior chamber in 28, iris in 24, pupil in 38 , lens in 15 , angle in 3 and posterior segment in 23 patients. Multiple ocular structure involvements were seen in the same patient.

\begin{tabular}{|c|c|c|}
\hline Ocular Structure & No. of Patients & \% Age \\
\hline Periorbital Lesions & 41 & 41.0 \\
\hline Eyelid & 66 & 66.0 \\
\hline Conjunctiva & 79 & 79.0 \\
\hline Cornea & 43 & 43.0 \\
\hline Anterior Chamber & 28 & 28.0 \\
\hline Iris & 24 & 22.0 \\
\hline Pupil & 38 & 39.0 \\
\hline Lens & 15 & 17.0 \\
\hline Angle & 3 & 3.0 \\
\hline Posterior Segment & 23 & 23.0 \\
\hline \multicolumn{2}{|c|}{ Table 9. Showing Trauma to Ocular Structures } \\
\hline \multicolumn{2}{|c}{}
\end{tabular}

\begin{tabular}{|c|c|c|}
\hline Periorbital Lesion & No. of Patients & \% Age \\
\hline Oedema & 32 & 78.0 \\
\hline Laceration & 8 & 19.5 \\
\hline Abrasion & 7 & 14.6 \\
\hline Burns & 3 & 7.3 \\
\hline Ecchymosis & 2 & 4.8 \\
\hline \multicolumn{2}{|c|}{ Total } & $\mathbf{4 1}$ \\
\hline \multicolumn{2}{|c|}{ Table 10. Showing Periorbital Lesion } \\
\hline
\end{tabular}

Table 10 shows that out of 41 periorbital lesions, commonest was oedema seen in 32 patients followed by laceration ( 8 patients), abrasion (7 patients), burns (3 patients) and ecchymosis ( 2 patients).

Table 11 shows that out of 66 eye lid injuries, oedema (62 patients) was the most common finding followed by ecchymosis (53 patients), laceration (14 patients), burns ( 3 patients), ptosis ( 2 patients) and scar ( 1 patient). Multiple types of eyelid injuries were seen in the same patient.

\begin{tabular}{|c|c|c|}
\hline Eyelid & No. of Patients & \% Age \\
\hline Oedema & 62 & 93.9 \\
\hline Ecchymosis & 53 & 80.3 \\
\hline Laceration & 14 & 21.2 \\
\hline Burns & 3 & 4.5 \\
\hline Ptosis & 2 & 3.0 \\
\hline Scar & 1 & 1.5 \\
\hline Total & $\mathbf{6 6}$ & \\
\hline \multicolumn{2}{|c|}{ Table 11. Showing Eyelid Involvement } \\
\hline
\end{tabular}

\begin{tabular}{|c|c|c|}
\hline Conjunctiva & No. of Patients & $\%$ Age \\
\hline Chemosis & 61 & 77.2 \\
\hline $\begin{array}{c}\text { Subconjunctival } \\
\text { Haemorrhage }\end{array}$ & 51 & 64.6 \\
\hline Congestion & 28 & 35.4 \\
\hline Laceration & 10 & 12.66 \\
\hline Total & 79 & \\
\hline \multicolumn{3}{|c|}{ Table 12. Showing Conjunctival Lesions } \\
\hline
\end{tabular}

Table 12 shows that out of 100 patients, 79 had conjunctival injuries. Multiple types of conjunctival injuries 
were found in the same patient. Chemosis (61 patients) was the most common finding followed by subconjunctival haemorrhage (51 patients), congestion (28 patients) and laceration in 10 patients.

\begin{tabular}{|c|c|c|}
\hline Corneal & No. of Patients & \% Age \\
\hline Oedema & 19 & 44.1 \\
\hline Laceration & 17 & 39.5 \\
\hline Opacity & 10 & 23.3 \\
\hline Abrasion & 10 & 23.3 \\
\hline Ulceration & 7 & 1.6 \\
\hline PEE & 1 & 2.3 \\
\hline Total & $\mathbf{4 3}$ & \\
\hline \multicolumn{2}{|c|}{ Table 13. Showing Corneal Involvement }
\end{tabular}

Table 13 shows that out of 43 patients with corneal injuries, 19 patients had corneal oedema, 17 had lacerated cornea, 10 each had opacity and abrasion, 7 had corneal ulceration, 1 had punctate epithelial erosions. Multiple types of corneal injuries were found in the same patient.

\begin{tabular}{|c|c|c|}
\hline Anterior Chamber & No. of Patients & \% Age \\
\hline Collapse & 19 & 67.85 \\
\hline Irregular & 7 & 25 \\
\hline Hyphaema & 7 & 25.0 \\
\hline Uveitis & 5 & 17.8 \\
\hline Foreign Body & 1 & 3.5 \\
\hline \multicolumn{2}{|c|}{ Total } & $\mathbf{2 8}$ \\
\hline Table 14. Showing Anterior Chamber (AC) Involvement
\end{tabular}

Table 14 shows that out of 28 patients with injury involving the anterior chamber, 19 had collapsed AC, 7 each had irregular AC and hyphaema, 5 had uveitis and 1 had foreign body. Multiple anterior chamber lesions were found in the same patient.

\begin{tabular}{|c|c|c|}
\hline Iris & No. of Patients & \% Age \\
\hline Prolapse & 11 & 45.8 \\
\hline Sphincter Tear & 6 & 25.0 \\
\hline Iridodialysis & 4 & 16.7 \\
\hline Iridodonesis & 2 & 8.3 \\
\hline Laceration & 1 & 41.7 \\
\hline Not Accessible (NA) & 1 & \\
\hline \multicolumn{2}{|c|}{ Total } & $\mathbf{2 4}$ \\
\hline \multicolumn{2}{|l}{ Table 15. Showing Iris Involvement } \\
\hline
\end{tabular}

Table 15 shows that out of 24 with iris injury, 11 had iris prolapse, 6 had pupillary sphincter tear, 4 had iridodialysis, 2 had iridodonesis and 1 had a laceration, whereas 1 could not be assessed. Multiple types of iris injuries were observed in the same patient.

\begin{tabular}{|c|c|c|}
\hline Pupil & No. of Patients & \% Age \\
\hline Irregular & 15 & 39.5 \\
\hline Eccentric & 15 & 39.5 \\
\hline Mydriatic & 8 & 21.1 \\
\hline Sluggish & 7 & 18.4 \\
\hline RAPD & 3 & 7.9 \\
\hline Posterior Synechiae & 2 & 5.3 \\
\hline
\end{tabular}

\begin{tabular}{|c|c|c|}
\hline NA & 1 & \\
\hline Total & $\mathbf{3 8}$ & \\
\hline \multicolumn{2}{|c|}{ Table 16. Showing Pupil Involvement } \\
\hline
\end{tabular}

Table 16 shows that out of 38 patients with trauma to pupil, 15 each had irregular and eccentric pupil, 8 had mydriasis, 4 had a sluggish reaction, 3 had RAPD (Relative Afferent Pupillary Defect) and 2 had posterior synechiae whereas 1 could not be assessed. Multiple types of pupillary lesions were encountered in the same patient.

\begin{tabular}{|c|c|c|}
\hline Sclera & No. of Patients & \% Age \\
\hline Laceration & 9 & 81.8 \\
\hline Rupture & 2 & 18.2 \\
\hline Total & $\mathbf{1 1}$ & \\
\hline \multicolumn{2}{|c|}{ Table 17. Showing Scleral Involvement } \\
\hline
\end{tabular}

Table 17 shows that out of 100 patients, 9 had a laceration of sclera and 2 had ruptured sclera.

\begin{tabular}{|c|c|c|}
\hline Lens & No. of Patients & \% Age \\
\hline Cataract & 11 & 73.3 \\
\hline Subluxation & 3 & 6.7 \\
\hline Dislocation & 2 & 13.33 \\
\hline Could not be assessed & 1 & 6.6 \\
\hline Total & $\mathbf{1 5}$ & \\
\hline \multicolumn{2}{|c|}{ Table 18. Showing Injury to the Lens } \\
\hline
\end{tabular}

Table 18 shows that 15 out of 100 patients had lenticular injury, in which 11 had cataract, 3 had subluxation, 2 had dislocation and it could not be assessed in 1 patient. Multiple lenticular injuries were seen in the same patient.

\begin{tabular}{|c|c|c|}
\hline Angle & No. of Patients & \% Age \\
\hline Recession & 3 & 3.0 \\
\hline Normal & 74 & 74.0 \\
\hline NA & 23 & 23.0 \\
\hline \multicolumn{2}{|c|}{ Table 19. Showing Angle Involvement } \\
\hline
\end{tabular}

Table 19 shows that out of 100 patients 3 had an angle recession, whereas it could not be assessed in 23 patients.

Table 20 shows that 9 patients out of 100 suffered traumatic effect to vitreous, of which 6 had haemorrhage, 4 had PVD (Posterior Vitreous Detachment) and 2 had vitreous prolapse. Multiple vitreous lesions were found in the same patient.

\begin{tabular}{|c|c|c|}
\hline Vitreous & No. of Patients & \% Age \\
\hline Haemorrhage & 6 & 66.7 \\
\hline PVD & 4 & 44.4 \\
\hline Vitreous Prolapse & 2 & 22.2 \\
\hline Total & 9 & \\
\hline \multicolumn{2}{|c|}{ Table 20. Showing Vitreous Involvement } \\
\hline
\end{tabular}

Table 21 shows that out of 12 patients with retinal injury, 5 had retinal detachment followed by commotio retinae in 3 patients, optic atrophy in 2 patients and 1 patient each had tear and macular hole.

\begin{tabular}{|c|c|c|}
\hline Retina & No. of Patients & \% Age \\
\hline Retinal Detachment & 5 & 41.7 \\
\hline Commotio Retinae & 3 & 25.0 \\
\hline Optic Atrophy & 2 & 16.7 \\
\hline
\end{tabular}




\begin{tabular}{|c|c|c|}
\hline Tear & 1 & 8.3 \\
\hline Macular Hole & 1 & 8.3 \\
\hline Total & $\mathbf{1 2}$ & \\
\hline \multicolumn{2}{|c|}{ Table 21. Showing Trauma to Retina } \\
\hline
\end{tabular}

\begin{tabular}{|c|c|c|}
\hline Choroid & No. of Patients & \% Age \\
\hline Rupture & 2 & 66.7 \\
\hline Laceration & 1 & 33.3 \\
\hline Total & 3 & \\
\hline \multicolumn{2}{|c|}{ Table 22. Showing Choroidal Involvement } \\
\hline
\end{tabular}

Table 22 shows that out of 100 patients 2 suffered from choroidal rupture, whereas 1 had a laceration of choroid.

\begin{tabular}{|c|c|c|c|c|}
\hline $\begin{array}{c}\text { Time } \\
\text { Interval }\end{array}$ & \begin{tabular}{|c|} 
Presenting \\
VA
\end{tabular} & $\begin{array}{c}\text { No. of } \\
\text { Patients }\end{array}$ & $\begin{array}{l}\text { Final } \\
\text { BCVA }\end{array}$ & $\begin{array}{c}\text { No. of } \\
\text { Patients }\end{array}$ \\
\hline \multirow{4}{*}{$<24$ hours } & $6 / 6-6 / 18$ & 7 & $6 / 6-6 / 18$ & 11 \\
\hline & $6 / 24-6 / 60$ & 3 & $6 / 24-6 / 60$ & 0 \\
\hline & $5 / 60-3 / 60$ & 0 & $5 / 60-3 / 60$ & 0 \\
\hline & \begin{tabular}{|c|}
$<3 / 60$ \\
\end{tabular} & 1 & $<3 / 60$ & 0 \\
\hline \multirow{4}{*}{1 - 5 days } & $6 / 6-6 / 18$ & 28 & $6 / 6-6 / 18$ & 40 \\
\hline & $6 / 24-6 / 60$ & 12 & $6 / 24-6 / 60$ & 1 \\
\hline & $5 / 60-3 / 60$ & 0 & $5 / 60-3 / 60$ & 0 \\
\hline & $<3 / 60$ & 1 & $<3 / 60$ & 0 \\
\hline \multirow{4}{*}{6 - 30 days } & $6 / 6-6 / 18$ & 7 & $6 / 6-6 / 18$ & 10 \\
\hline & $6 / 24-6 / 60$ & 3 & $6 / 24-6 / 60$ & 5 \\
\hline & $5 / 60-3 / 60$ & 5 & $5 / 60-3 / 60$ & 1 \\
\hline & $<3 / 60$ & 2 & $<3 / 60$ & 1 \\
\hline \multirow{4}{*}{$\begin{array}{c}1-12 \\
\text { months }\end{array}$} & $6 / 6-6 / 18$ & 3 & $6 / 6-6 / 18$ & 3 \\
\hline & $6 / 24-6 / 60$ & 0 & $6 / 24-6 / 60$ & 3 \\
\hline & $5 / 60-3 / 60$ & 0 & $5 / 60-3 / 60$ & 0 \\
\hline & $<3 / 60$ & 6 & $<3 / 60$ & 3 \\
\hline \multirow{4}{*}{$\begin{array}{c}>12 \\
\text { months }\end{array}$} & $6 / 6-6 / 18$ & 0 & $6 / 6-6 / 18$ & 1 \\
\hline & $6 / 24-6 / 60$ & 0 & $6 / 24-6 / 60$ & 0 \\
\hline & $5 / 60-3 / 60$ & 1 & $5 / 60-3 / 60$ & 1 \\
\hline & $<3 / 60$ & 2 & $<3 / 60$ & 1 \\
\hline Total & & 81 & & 81 \\
\hline \multicolumn{5}{|c|}{$\begin{array}{l}\text { Table 23. Showing Correlation between Visual } \\
\text { Acuity and Time Interval between Injury and Presentation } \\
\text { to the Hospital in Patients with Closed Globe Injury }\end{array}$} \\
\hline
\end{tabular}

\begin{tabular}{|c|c|c|c|c|}
\hline $\begin{array}{c}\text { Time } \\
\text { Interval }\end{array}$ & $\begin{array}{c}\text { Presenting } \\
\text { VA }\end{array}$ & $\begin{array}{c}\text { No. of } \\
\text { Patients }\end{array}$ & $\begin{array}{l}\text { Final } \\
\text { BCVA }\end{array}$ & $\begin{array}{c}\text { No. of } \\
\text { Patients }\end{array}$ \\
\hline \multirow{4}{*}{$<24$ hours } & $6 / 6-6 / 18$ & 0 & $\begin{array}{l}6 / 6- \\
6 / 18\end{array}$ & 4 \\
\hline & $6 / 24-6 / 60$ & 1 & $\begin{array}{c}6 / 24- \\
6 / 60 \\
\end{array}$ & 7 \\
\hline & $5 / 60-3 / 60$ & 4 & $\begin{array}{l}5 / 60- \\
3 / 60\end{array}$ & 2 \\
\hline & $<3 / 60$ & 11 & $<3 / 60$ & 3 \\
\hline \multirow{4}{*}{1 - 5 days } & $6 / 6-6 / 18$ & 0 & $\begin{array}{l}6 / 6- \\
6 / 18\end{array}$ & 0 \\
\hline & $6 / 24-6 / 60$ & 0 & $\begin{array}{c}6 / 24- \\
6 / 60\end{array}$ & 2 \\
\hline & $5 / 60-3 / 60$ & 1 & $\begin{array}{c}5 / 60- \\
3 / 60\end{array}$ & 1 \\
\hline & $<3 / 60$ & 2 & $<3 / 60$ & 0 \\
\hline \multirow{3}{*}{6 - 30 days } & $6 / 6-6 / 18$ & 0 & $\begin{array}{l}6 / 6- \\
6 / 18\end{array}$ & 0 \\
\hline & $6 / 24-6 / 60$ & 0 & $\begin{array}{c}6 / 24- \\
6 / 60\end{array}$ & 0 \\
\hline & $5 / 60-3 / 60$ & 0 & $5 / 60-$ & 0 \\
\hline
\end{tabular}

\begin{tabular}{|c|c|c|c|c|}
\hline \multirow{4}{*}{$\begin{array}{c}1-12 \\
\text { months }\end{array}$} & $</ 6 / 60$ & 0 & $</ 60$ & \\
\cline { 2 - 5 } & $6 / 6-6 / 18$ & 0 & $\begin{array}{c}6 / 6- \\
6 / 18\end{array}$ & 0 \\
\cline { 2 - 5 } & $5 / 60-3 / 60$ & 0 & $\begin{array}{c}5 / 60- \\
3 / 60\end{array}$ & 0 \\
\cline { 2 - 5 } & $<3 / 60$ & 0 & $<3 / 60$ & 0 \\
\hline \multirow{4}{*}{$\begin{array}{c}6 \\
\text { months }\end{array}$} & $6 / 6-6 / 18$ & 0 & $\begin{array}{c}6 / 6- \\
6 / 18\end{array}$ & 0 \\
\cline { 2 - 5 } & $6 / 24-6 / 60$ & 0 & $\begin{array}{c}6 / 24- \\
6 / 60\end{array}$ & 0 \\
\cline { 2 - 5 } & $5 / 60-3 / 60$ & 0 & $\begin{array}{c}5 / 60- \\
3 / 60\end{array}$ & 0 \\
\cline { 2 - 5 } & $<3 / 60$ & 0 & $<3 / 60$ & 0 \\
\hline Total & & 19 & & 19 \\
\hline
\end{tabular}

Table 24. Showing Correlation between Visual Acuity and Time Interval between Injury and Presentation to the Hospital in Patients with Open Globe Injury

\begin{tabular}{|c|c|c|c|}
\hline Presenting Visual Acuity & $\begin{array}{c}\text { No. of } \\
\text { Patients }\end{array}$ & $\begin{array}{c}\text { Final } \\
\text { BCVA }\end{array}$ & $\begin{array}{c}\text { No. of } \\
\text { Patients }\end{array}$ \\
\hline $6 / 6-6 / 18$ & 45 & $6 / 6-6 / 18$ & 65 \\
\hline $6 / 24-6 / 60$ & 18 & $6 / 24-6 / 60$ & 9 \\
\hline $5 / 60-3 / 60$ & 6 & $5 / 60-3 / 60$ & 2 \\
\hline$<3 / 60$ & 12 & $<3 / 60$ & 5 \\
\hline Total & $\mathbf{8 1}$ & Total & $\mathbf{8 1}$ \\
\hline $\begin{array}{c}\text { Table 25. Showing Correlation between Visual } \\
\text { Acuity Outcome and Closed Globe Injury }\end{array}$ \\
\hline
\end{tabular}

Table 25 shows that out of 81 patients with closed globe injuries at the time of presentation, 45 had VA between $6 / 6$ 6/18, 18 had between 6/24 -6/60, 6 had between 5/60 $3 / 60$ and 12 had $<3 / 60$. However, after treatment 65 had BCVA between 6/6 - 6/18, 9 had between 6/24 - 6/60, 2 had between $5 / 60-3 / 60$ and only 5 had $<3 / 60$.

\begin{tabular}{|c|c|c|c|}
\hline $\begin{array}{c}\text { Presenting } \\
\text { Visual Acuity }\end{array}$ & $\begin{array}{c}\text { No. of } \\
\text { Patients }\end{array}$ & Final BCVA & $\begin{array}{c}\text { No. of } \\
\text { Patients }\end{array}$ \\
\hline $6 / 6-6 / 18$ & 0 & $6 / 6-6 / 18$ & 4 \\
\hline $6 / 24-6 / 60$ & 1 & $6 / 24-6 / 60$ & 9 \\
\hline $5 / 60-3 / 60$ & 5 & $5 / 60-3 / 60$ & 3 \\
\hline$<3 / 60$ & 13 & $<3 / 60$ & 3 \\
\hline Total & 19 & Total & 19 \\
\hline Table 26. Showing Correlation between Visual Acuity \\
Outcome and Open Globe Injury \\
\hline
\end{tabular}

Table 26 shows that out of 19 patients with open globe injuries at the time of presentation, 1 had VA between $6 / 24$ 6/60, 5 had between 5/60 - 3/60 and 13 had $<3 / 60$. However, after treatment 4 had final BCVA between $6 / 6$. $6 / 18,9$ had between $6 / 24-6 / 60$ and 3 each had visual acuity between $5 / 60-3 / 60$ and $<3 / 60$.

\begin{tabular}{|c|c|c|c|c|}
\hline $\begin{array}{c}\text { Extent of } \\
\text { Injury }\end{array}$ & $\begin{array}{c}\text { Presenting } \\
\text { VA }\end{array}$ & $\begin{array}{c}\text { No. of } \\
\text { Patients }\end{array}$ & $\begin{array}{c}\text { Final } \\
\text { BCVA }\end{array}$ & $\begin{array}{c}\text { No. of } \\
\text { Patients }\end{array}$ \\
\hline \multirow{3}{*}{$\begin{array}{c}\text { Anterior } \\
\text { Segment } \\
\text { (AS) }\end{array}$} & $6 / 6-6 / 18$ & 43 & $\begin{array}{c}6 / 6- \\
6 / 18\end{array}$ & 65 \\
\cline { 2 - 5 } & $6 / 24-6 / 60$ & 16 & $\begin{array}{c}6 / 24- \\
6 / 60\end{array}$ & 15 \\
\cline { 2 - 5 } & $5 / 60-3 / 60$ & 11 & $5 / 60-$ & 3 \\
\hline
\end{tabular}




\begin{tabular}{|c|c|c|c|c|}
\hline & & & $3 / 60$ & \\
\cline { 2 - 5 } & $<3 / 60$ & 14 & $<3 / 60$ & 1 \\
\hline \multirow{3}{*}{$\begin{array}{c}\text { Anterior } \\
\text { and } \\
\begin{array}{c}\text { Posterior } \\
\text { Segment }\end{array}\end{array}$} & $6 / 6-6 / 18$ & 2 & $\begin{array}{c}6 / 6- \\
6 / 18\end{array}$ & 4 \\
\cline { 2 - 5 } (AS + PS) & $5 / 60-3 / 60$ & 0 & $\begin{array}{c}5 / 60- \\
6 / 60\end{array}$ & 2 \\
\cline { 2 - 5 } & $<3 / 60$ & 11 & $<3 / 60$ & 7 \\
\hline $\begin{array}{c}\text { Total No. } \\
\text { of Patients }\end{array}$ & \multicolumn{2}{|c|}{100} & 100 \\
\hline Table 27. Showing Correlation between Extent of Injury \\
and Visual Acuity
\end{tabular}

Table 27 shows that out of 84 patients with anterior segment injury, 43 had initial VA between $6 / 6-6 / 18,16$ had between 6/24 -6/60, 11 had between 5/60 - 3/60 and 14 had $<3 / 60$. However, after treatment 65 had BCVA between $6 / 6-6 / 18,15$ had between $6 / 24-6 / 60,3$ had between $5 / 60$ - 3/60 and 1 had <3/60. Out of 16 patients with both anterior and posterior segment injury, 2 had initial VA between $6 / 6-6 / 18,3$ had between $6 / 24-6 / 60$, none had between $5 / 60-3 / 60$ and 11 had $<3 / 60$. However, after treatment, 4 had BCVA between $6 / 6-6 / 18,3$ had between $6 / 24-6 / 60,2$ had between $5 / 60-3 / 60$ and 7 had $<3 / 60(P$ $<0.05$, Pearson's Chi-squared test).

\begin{tabular}{|c|c|c|c|c|c|}
\hline $\begin{array}{c}\text { Initial } \\
\text { VA }\end{array}$ & No. & $\begin{array}{c}\text { \% } \\
\text { Age }\end{array}$ & $\begin{array}{c}\text { Final } \\
\text { BCVA }\end{array}$ & No. & $\begin{array}{c}\% \\
\text { Age }\end{array}$ \\
\hline $6 / 6-6 / 18$ & 45 & 45.0 & $6 / 6-6 / 18$ & 69 & 69.0 \\
\hline $6 / 24-6 / 60$ & 19 & 20.0 & $6 / 24-6 / 60$ & 18 & 18.0 \\
\hline $5 / 60-3 / 60$ & 11 & 11.0 & $5 / 60-3 / 60$ & 5 & 5.0 \\
\hline$<3 / 60$ & 25 & 25.0 & $<3 / 60$ & 8 & 8.0 \\
\hline \multicolumn{6}{|c|}{ Table 28. Showing Best Corrected Visual Acuity (BCVA) } \\
\hline
\end{tabular}

Table 28 shows that out of 100 patients, at the time of presentation 45 had VA between $6 / 6-6 / 18,19$ had between $6 / 24-6 / 60,11$ had between 5/60 - 3/60 and 25 had $<3 / 60$. However, after treatment 69 had BCVA between $6 / 6-6 / 18$, 18 had between $6 / 24-6 / 60,5$ had between $5 / 60-3 / 60$ and 8 had $<3 / 60$. Initial VA correlated with the final BCVA (Spearman's correlation coefficient $=0.658 ; \mathrm{P}<0.05$ ).

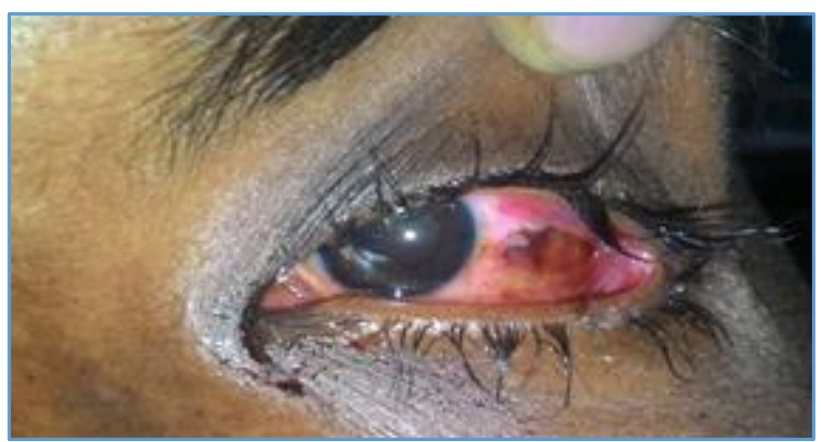

Table/Fig. 1: Conjunctival Laceration

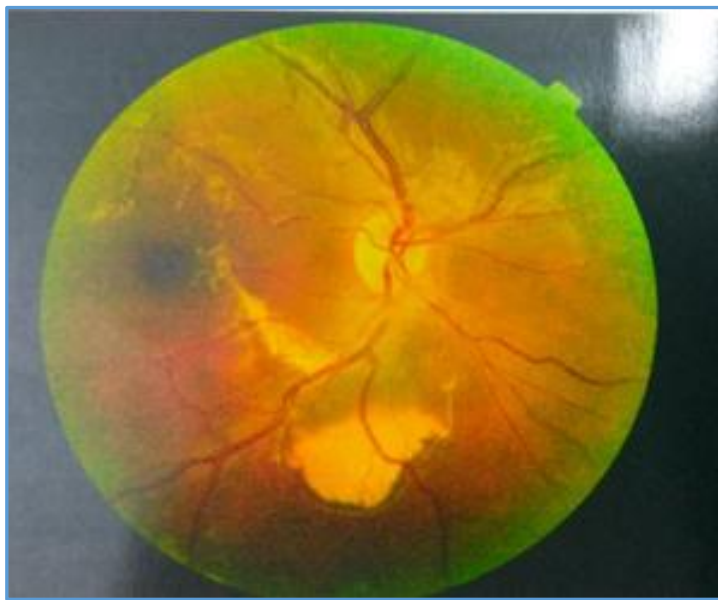

Table/Fig. 2: Fundus Photograph showing Choroidal Rupture

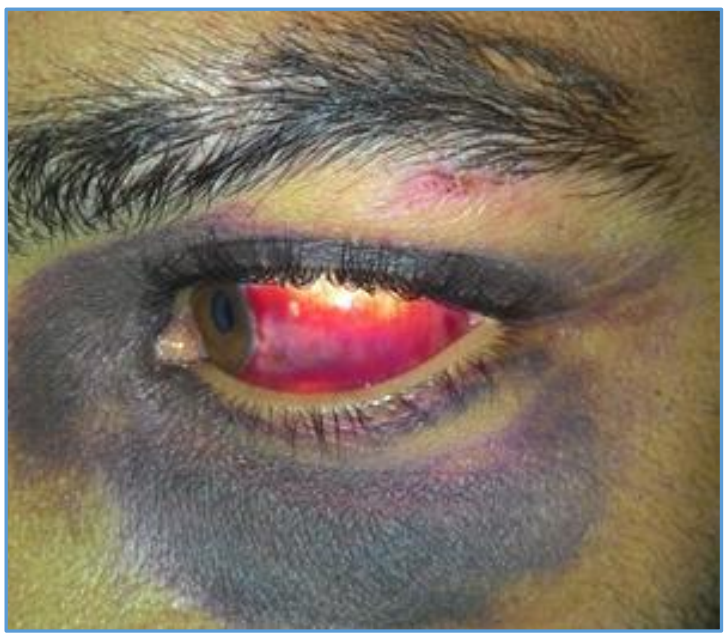

Table/Fig. 3: Lid Oedema with Ecchymosis and Subconjunctival Haemorrhage

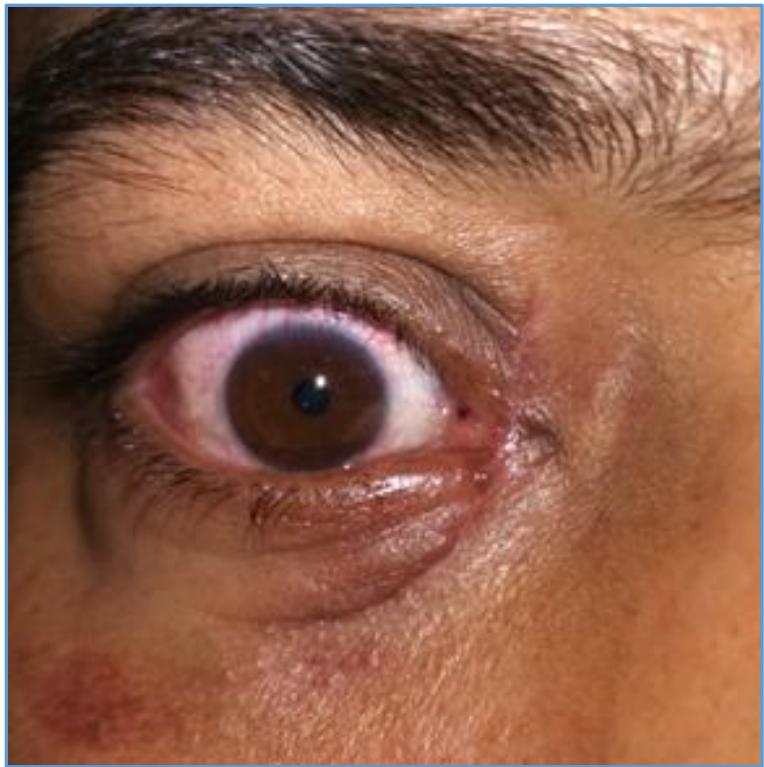

Table/Fig. 4: Lower Canaliculi Injury due to Blunt Trauma 


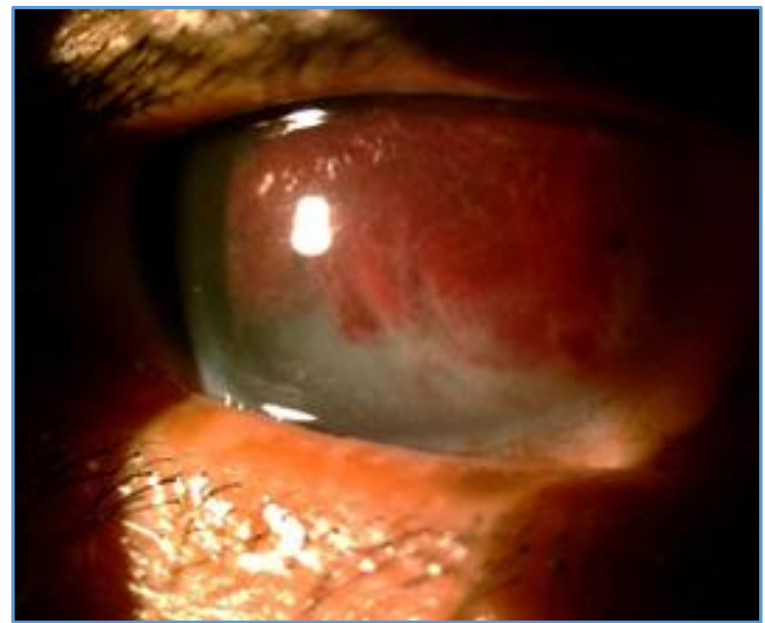

Table/Fig. 5: Traumatic Hyphaema and Cataract

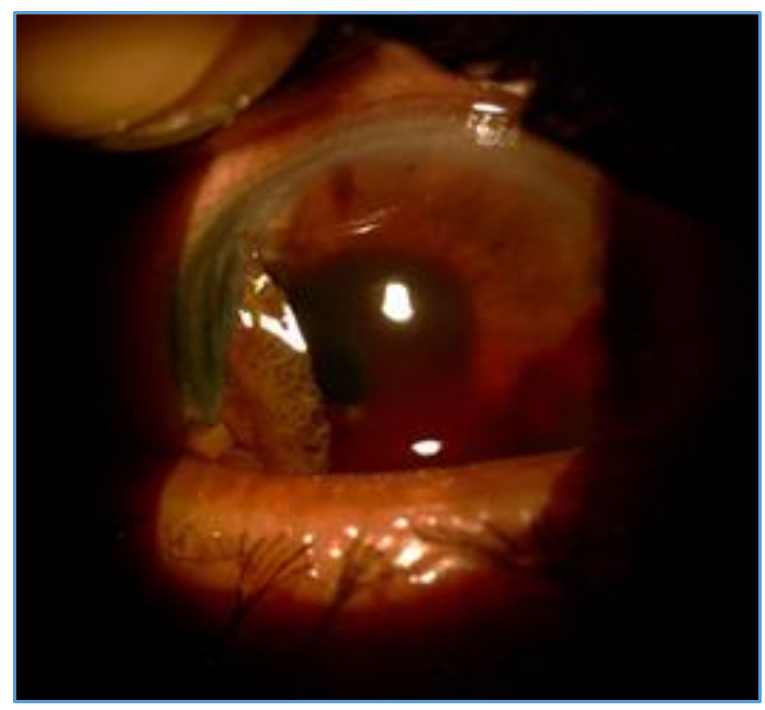

Table/Fig. 6: Corneal Laceration with Iris Prolapse and Hyphaema

Table/Fig. 7: Perforating Injury

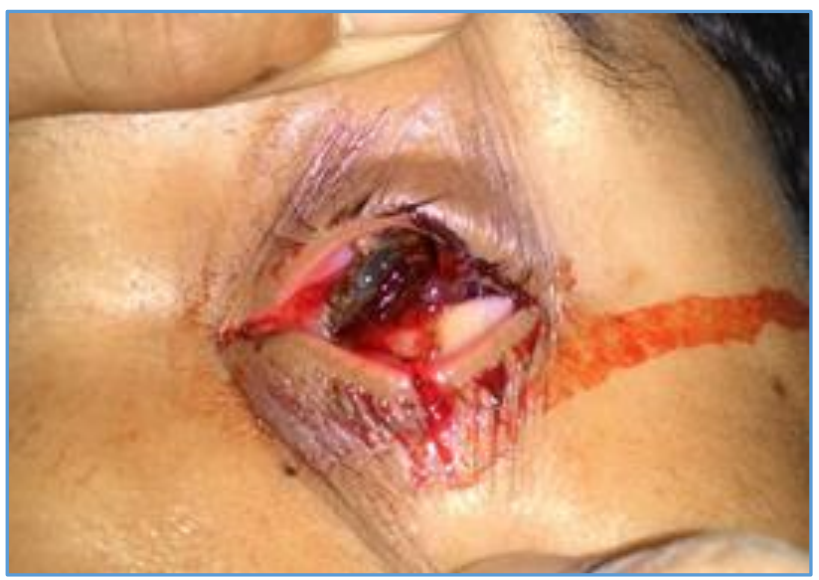

7(a): Open Globe Injury

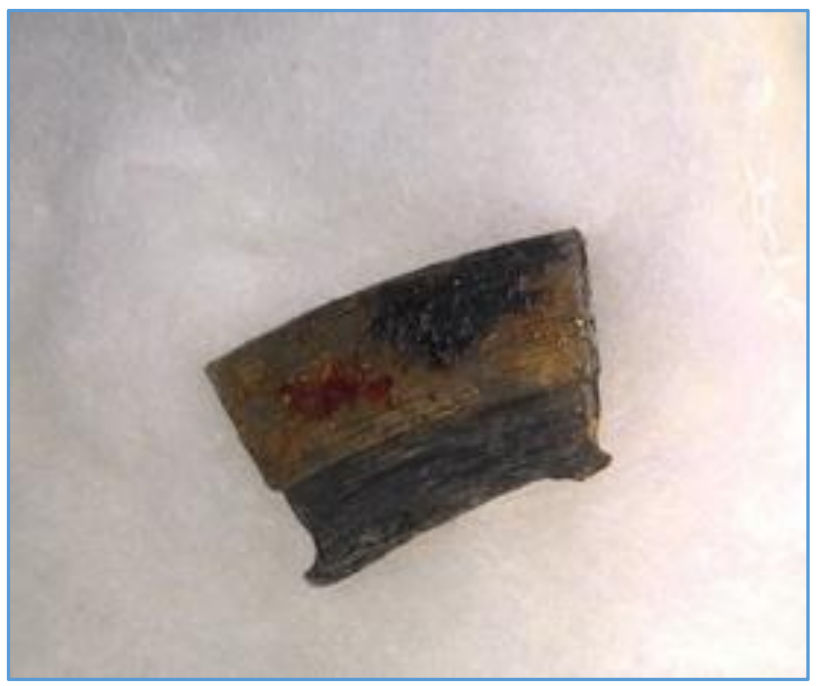

7(b): Metallic Blade retrieved from Eye

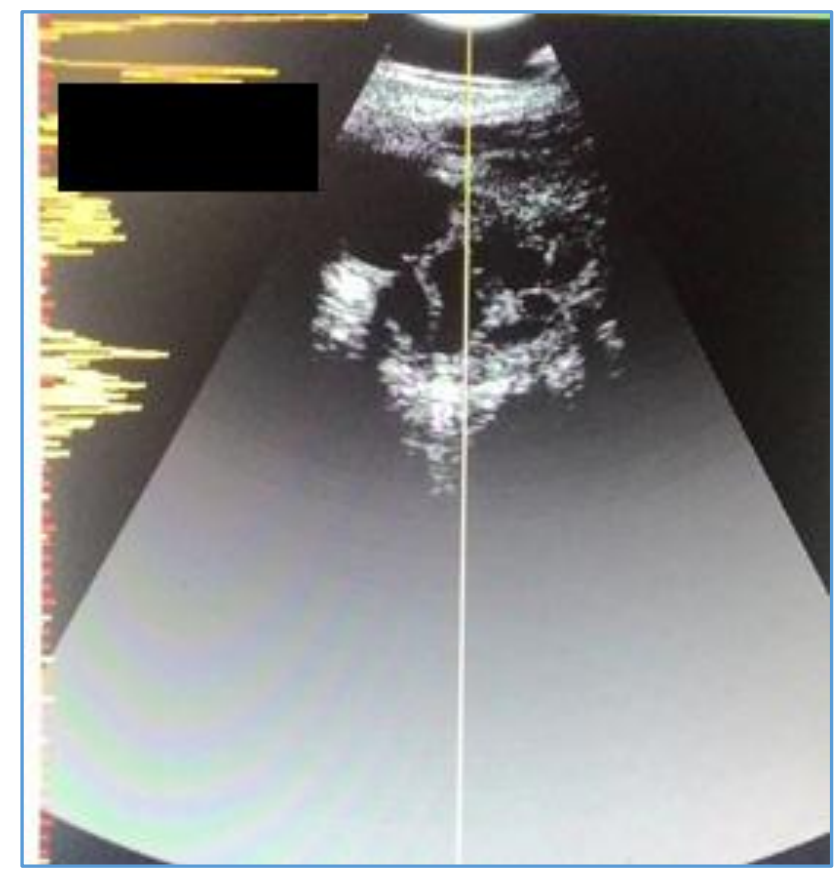

7(c): B Scan showing Posterior Segment Tear

\section{DISCUSSION}

In our study there was increased incidence of ocular injuries in younger age group and children, $65 \%$ patients being under 35 years of age. This was in close relation with Dhasmana $\mathrm{R}$ et $\mathrm{al}^{(8)}$ and Gupta $\mathrm{S}$ et al.(9)

Males were more commonly involved constituting $88 \%$ of the study group. Male preponderance is seen, because they are more frequently exposed to outdoor work compared to females. This was in correlation with Balaghafari A et al ${ }^{10}$ and Sharma AK et al.(11)

The most common cause of injury was road traffic accident $(30.0 \%)$ followed by injury due to wood $(12.0 \%)$ and stick $(10.0 \%)$. This is consistent with some studies, while others do have variety of findings. Enock ME et al(12) and Mowatt L et al(4) showed that motorcycle-related road traffic accident was the most common cause of ocular injury.

In our study $27 \%$ presented within 24 hours, whereas 44\% presented between 1 - 5 days. This was correlated with Saxena R et al.(13) 
Patients who came late after injury usually did not suffer from significant symptoms and signs at the time of injury or had received treatment elsewhere and were presenting now with sequelae or complications.

$81 \%$ had closed globe injuries, whereas $19 \%$ had open globe injuries. This was in close correlation with Nirmalan PK et al(14) and Pandita A.(15)

In our study, conjunctiva (79\%) was the commonly involved structure in ocular trauma followed by trauma to eyelid (66.0\%) and cornea (43\%).

Oedema (32.0\%) was the most common finding in periorbit, whereas oedema (62.0\%) followed by ecchymosis (53\%) were common in eyelid injuries. Conjunctival chemosis $(61 \%)$ followed by subconjunctival haemorrhage (51\%) were frequent injuries in conjunctiva. Oedema (19\%) and laceration $(17 \%)$ in cornea, collapsed anterior chamber $(19.0 \%)$ and hyphaema $(7.0 \%)$, prolapse $(11.0 \%)$ and sphincter tear $(6.0 \%)$ were associated frequently with trauma to iris, whereas irregular and eccentric pupil $(15.0 \%$ each), cataract $(11.0 \%)$, vitreous haemorrhage $(6.0 \%)$, retinal detachment $(5.0 \%)$, choroidal rupture $(2.0 \%)$ and scleral laceration $(9.0 \%)$ were the common injuries encountered in our study.

Enock ME et al(12) showed that the conjunctiva, lids and cornea were the mostly commonly affected structures during ocular trauma. Similarly, Pai SG et al(16) found that the most commonly involved structure was conjunctiva followed by lid and adnexa.

Out of 100 patients studied, 45 had VA between $6 / 18$ 6/6, 19 had between 6/24 - 6/60, 11 had between 5/60 $3 / 60$ and 25 had $<3 / 60$ at the time of presentation. However, after treatment, 69 had VA between $6 / 18$ - 6/6, 18 had between $6 / 24-6 / 60,5$ had between $5 / 60-3 / 60$ and 8 had < $3 / 60, p=0.000$ which is highly significant.

In a study by Qi Y et al,(17) it was concluded that $65.5 \%$ of patients achieved VA at $>20 / 60$. The factors associated with the final visual outcome were initial VA, injury type, wound location, the way of cataract removal and IOL implantation.

In the study by Maurya RP et al,(18) initial visual acuity was $\operatorname{good}(>/=6 / 18)$ in $36.59 \%$, moderate vision $(<6 / 18$ $6 / 60)$ in $26.83 \%$ and poor vision $(<6 / 60)$ in $21.95 \%$, while assessment was not possible in $14.63 \%$. Final visual acuity was $6 / 18$ or better in $46.34 \%$, between $<6 / 18$ to $3 / 60$ in $21.95 \%$ and $<3 / 60$ to no PL in $19.51 \%$. Final visual acuity could not be evaluated in $12.20 \%$ cases.

\section{CONCLUSION}

We conclude that ocular trauma is more common in males in younger age group. Majority present within 5 days of trauma. Road traffic accident is the most common cause of injury. Closed globe injuries are more common than open globe. Conjunctiva is the most frequently injured structure followed by eyelid. Post management visual acuity depends not only on severity of injury, but also on the time of presentation. Health education in schools and factories can help prevent and early manage ocular trauma. Prompt evaluation and treatment helps salvage the eye both anatomically and functionally, thus significantly reduce both social and economic burden on the society.

Interdisciplinary approaches and community-based strategies are important to make progress in this area of study to save vision.

\section{REFERENCES}

[1] Sethi S, Khan MD. Childhood Ocular Trauma. J Postgrad Med Inst Peshawar 2011;15(1):51-5.

[2] Mishra A, Parihar J, Verma A, et al. The pattern and visual outcomes of ocular trauma in a large zonal hospital in a non-operational role: A 36 months retrospective analysis. J Clin Ophthalmol Res 2014;2(3):141-4.

[3] Négrel AD, Thylefors B. The global impact of eye injuries. Ophthalmic Epidemiol 1998;5(3):143-69.

[4] Mowatt L, McDonald A, Ferron-Boothe D. Hospitalization trends in adult ocular trauma at the University Hospital of the West Indies. West Indian Med J 2012;61(6):605-9.

[5] Misra S, Nandwani R, Gogri P, et al. Clinical profile and visual outcome of ocular injuries in a rural area of western India. Australas Med J 2013;6(11):560-4.

[6] Canavan YM, O'Flaherty MJ, Archer DB, et al. A 10-year survey of eye injuries in northern Ireland, 1967-76. Br J Ophthalmol 1980;64(8):618-25.

[7] Kinderan YV, Shrestha E, Maharjan IM, et al. Pattern of ocular trauma in the western region of Nepal. Nepal J Ophthalmol 2012;4(1):5-9.

[8] Dhasmana R, Bahadur H, Jain K. Profile of ocular trauma in Uttarakhand, a hospital based study. Indian J Community Health 2013;24(4):297-303.

[9] Gupta S, Malhotra AK, Gehra A, et al. Pattern of ocular trauma at MLB medical college, Jhansi (UP)-a retrospective study. Int $\mathrm{J}$ Biomed $\mathrm{Adv}$ Res 2015;6(8):589-93.

[10] Balaghafari A, Siamian H, Aligolbandi K. Ocular trauma: 2 years retrospective study in Sari, Iran. Mater Sociomedica 2013;25(4):230-2.

[11] Sharma AK, Aslami AN, Srivastava JP, et al. Visual outcome of traumatic cataract at a tertiary eye care centre in north India: a prospective study. J Clin Diagn Res 2016;10(1):NC05-08.

[12] Enock ME, Omoti AE, Alikah AA. Risk factors identification for ocular trauma in patients who presented in a suburban tertiary care hospital in Nigeria. Saudi Med J 2007;28(9):1385-8.

[13] Saxena R, Sinha R, Purohit A, et al. Pattern of pediatric ocular trauma in India. Indian $\mathrm{J}$ Pediatr 2002;69(10):863-7.

[14] Nirmalan PK, Katz J, Tielsch JM, et al. Ocular trauma in a rural south Indian population: the Aravind comprehensive eye survey. Ophthalmology 2004;111(9):1778-81.

[15] Pandita A, Merriman M. Ocular trauma epidemiology: 10-year retrospective study. New Zealand Med J 2012;125(1348):61-9.

[16] Pai SG, Kamath SJ, D’Souza S, et al. A Clinical study of blunt ocular trauma in a tertiary care centre. Online Journal of Health and Allied Sciences 2013;12(2):10-3.

[17] Qi Y, Zhang YF, Zhu Y, et al. Prognostic factors for visual outcome in traumatic cataract patients. J Ophthalmol 2016;2016:e1748583.

[18] Maurya RP, Singh VP, Yadav I, et al. Profile of pediatric ocular trauma at a tertiary eye care centre in northern India. Indian Journal of Clinical and Experimental Ophthalmology 2015;1(2):73-6. 